\title{
Construction of 3D Interactive Virtual Simulation Experiment Platform for Coal Spontaneous Combustion
}

\author{
Pengtao Jia, Zhilong Zhao, Jun Deng
}

\begin{abstract}
In order to predict the risk of spontaneous combustion of coal, we construct a 3D interactive virtual simulation experiment platform. The platform combines with the physical experiment device and theory of coal spontaneous combustion. The platform can not only visualize the physical experiment process, calculate key parameters, help establish numerical models, but also visualize the numerical simulation process. The experimenter can also modify the numerical model and visualize the result of the spontaneous combustion process of the modified coal on this platform. The platform combines the advantages of physical simulation and numerical simulation, which saves the time of the researchers and promotes the research of coal spontaneous combustion mechanism.
\end{abstract}

Index Terms-Predict, Virtual Reality, Interactive, Coal Spontaneous Combustion.

\section{INTRODUCTION}

The goaf is the part of a mine from which the mineral has been partially or wholly removed. It is the main place of coal spontaneous combustion, which directly threatens the safety of the coal mine production, resulting in a large amount of coal resources loss and "freezing". Due to the concealment and uncertainty of the location of high temperature in the mined area, it is easy to lead to lagging behind in finding and taking measures, resulting in a waste of manpower and material resources, which brings hidden danger to mine safety production. Therefore, the spontaneous combustion of coal in the goaf is a difficult problem to be solved.

In order to predict the risk of spontaneous combustion of coal, determination of spontaneous combustion dangerous region, many scholars put forward a lot of mathematical and physical models to simulate coal spontaneous combustion. Some scholars, such as Schmal Dick [1], Brooks Kevin [2],

Pengtao Jia received the Ph.D. degree from the Department of Computer Science, Northwestern Polytechnical University, Xian, China, in 2008. She is currently a Professor with the Department of Computer Science, Xi'an University of Science and Technology, China. Her current research interests include Machine learning, data mining,visualization of coal mine safety.

Zhilong Zhao is currently pursuing the master's degree with the Department of Computer Science, Xi'an University of Science and Technology, China.

Jun Deng is currently a Professor with the Department of Safety Scienc e and Engineering, Xi' an University of Science and Technology, China. He is the director of Key Laboratory of Western Mine Exploitation and Hazar d Prevention of Ministry of Education.
Zhang Juwei [3], Lei Changkun [4], Liu Wei [5], established mathematical models based on the reactor, heat and mass transfer and Arrhenius formula to simulate the process of coal spontaneous combustion. Some other scholars, such as $\mathrm{Yu}$ Minggao, et al [6], Liang Yuntao [7], Wang Deming [8], Yu Zhijin [9], Liu wei [10], Xu Jingcai, Wen Hu, Deng Jun, et al [11-12], established the physical models based on coal oxidation theory. In summary, the research of coal spontaneous combustion is divided into two aspects: numerical simulation and physical simulation. The advantages of numerical simulation are low cost and short period. The drawback is that the model is complex and difficult to get the analytical solution. The core parameters of the coal spontaneous combustion process (the heat release parameter) are calculated by the theoretical method. The other key parameters in the model are empirical formulas, and the simulation results are different from the actual situation. The advantage of physical simulation is that it can simulate the condition of spontaneous of underground coal better. The drawback is that the amount of coal used in the experiment is large, the cycle is long and the cost is high, so the experimental process cannot be directly displayed.

If we can combine the advantages of physical and numerical simulation, the core parameters obtain by physical experiment of the coal sample amount. Then based on the theory of coal oxygen complex interaction, the numerical model of coal spontaneous combustion process is established on the basis of porous media seepage mechanics and heat and mass transfer theory. The virtual simulation test platform is constructed by using virtual reality technology. After validating and correcting the numerical model on the virtual simulation platform, and then applying the numerical model to the worksite, it can set up a better prediction model for the spontaneous combustion risk area in a short time.

Therefore, we constructed a 3D interactive virtual simulation platform based on a large number of experimental data and physical device of innovation team of Xi'an University of Science and Technology.

\section{PhysiCAL DEVICE}

In order to simulate the whole process of coal spontaneous combustion, an experiment system of coal spontaneous combustion has been developed by Xi'an University of Science and Technology. The experimental device simulates the initial temperature, humidity and heat storage conditions of the downhole, and the air compressor is used to supply the air volume to simulate the actual air leakage condition. The 
schematic diagram and diagram of the coal spontaneous combustion experiment system are shown in Fig. 1. In order to effectively monitor and deal with the experiment, the hardware system structure of the $3 \mathrm{D}$ virtual simulation experiment platform of coal spontaneous combustion is designed, as shown in Fig. 2.

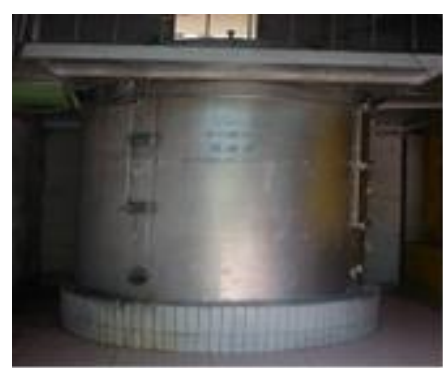

Fig. 1a. The appearance of physical device.

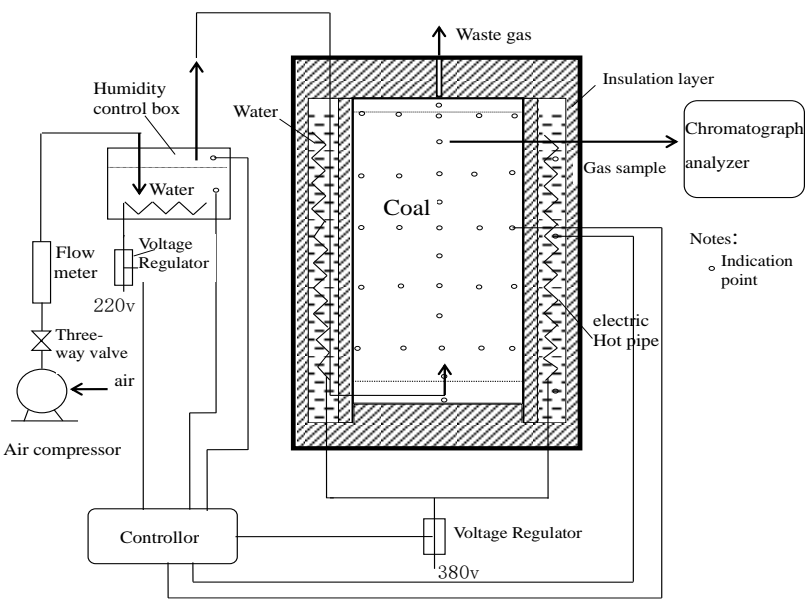

Fig. 1b. The schematic diagram of the coal spontaneous combustion experiment system.

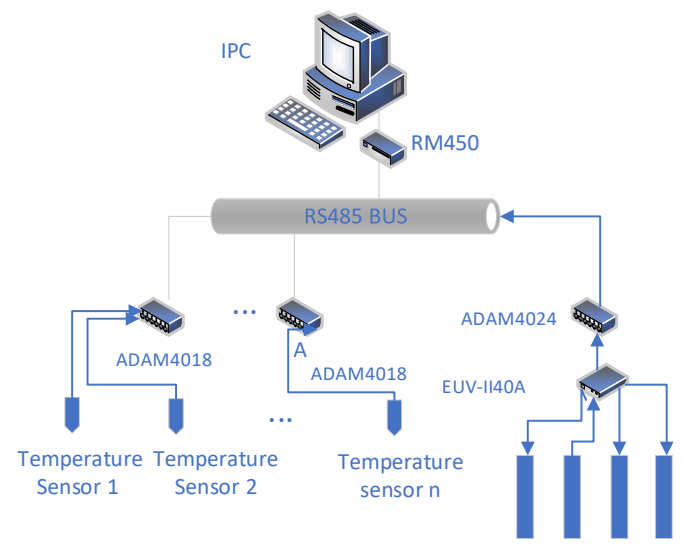

Heating rod

Fig. 2. The hardware of the coal spontaneous combustion experiment system.

In Fig.2, Industrial Personal Computer (IPC) with 3D virtual simulation platform of coal spontaneous combustion is the center of the data fusion processing, database management and temperature monitoring center. IPC equipped with RS232-RS485 conversion module RM450; ADAM4018 remote temperature sensor acquisition module, can collect 8 temperature sensor signals, and converts it into the corresponding temperature value ADAM4024 for output. The control module can output 4 voltage signals. EUV II40A is a single-phase solid voltage module is used to achieve control of weak to strong power, the input voltage level to control the output power of the heating rod.

In XKII, experimental furnace loading capacity of $2.5 \mathrm{t}$ as an example, in the inside and outside of the furnace in different position and direction were arranged for 140 temperature sensors, including 130 road in coal samples, 4 in water, 4 is the ambient temperature around the furnace body, 2 in the inlet end, respectively, for coal temperature the monitoring of furnace water temperature, ambient temperature and air temperature data. The ADAM4018 module will collect the temperature value through the RS485 bus channel to the RM450 communication conversion module, send it to the industrial control computer for error processing, get the consistent measurement results, and then do data fusion. Once after the integration of coal temperature and the water temperature of the temperature difference exceeds the preset value, the IPC output voltage control module ADAM4024 conversion module through the RM450 communication, and then through the heat heating EUV II40A control layout in water pipes in the coal temperature and temperature difference in temperature maintained in the range given in advance in order to ensure the coal, almost no heat loss to the outside world, to simulate the underground coal spontaneous combustion oxidation heating purposes.

\section{ViRtual Simulation SyStem of COAL SPONTANEOUS COMBUSTION}

The virtual simulation system of coal spontaneous combustion has five modules. They are project management module, monitoring data acquisition, virtual simulation, numerical calculation and data management.

\section{A. Design of 3D Model Figures}

The three dimensional model should be built on the basis of realistic objects, so that we can restore the real things in virtual terms, and the degree of simulation is high, so that we can feel the true feelings of users. The platform is based on Visual C++ environment and OpenGL 3D graphics library. The principle of modeling is to maximize the resource consumption of the system when invoking the model.

The model of the experiment furnace is shown in Fig. 3. The whole model of the laboratory and goaf are shown in Fig. 4.
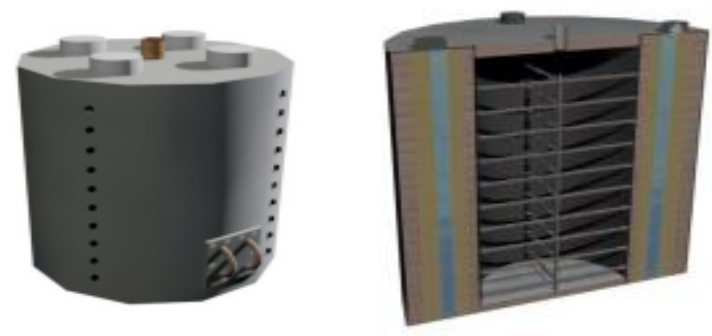

Fig. 3. The model of the experiment furnace. 


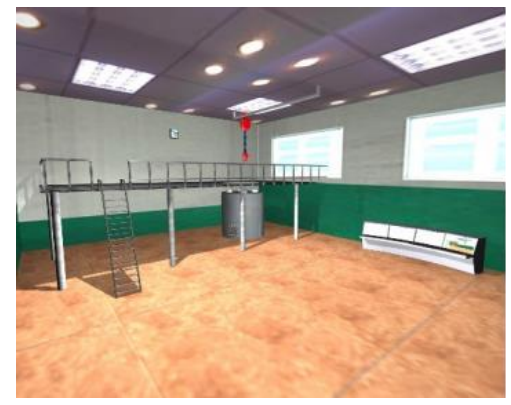

Fig. 4a. The whole model of the laboratory.

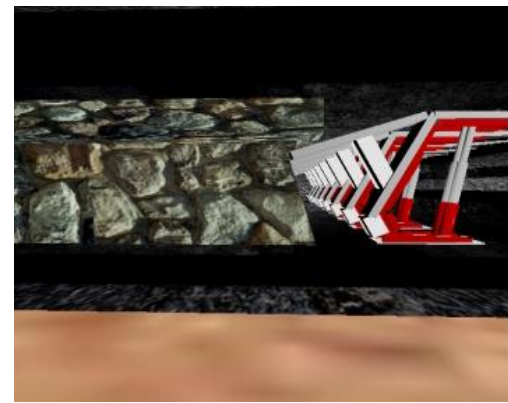

Fig. 4b. The whole model of the goaf.

\section{B. Monitoring and Data Acquisition Module}

This module mainly collects data of physical experiment system, including four functions: data acquisition, data query, data correction, water temperature control. The monitoring and data acquisition interface is shown in Fig. 5.

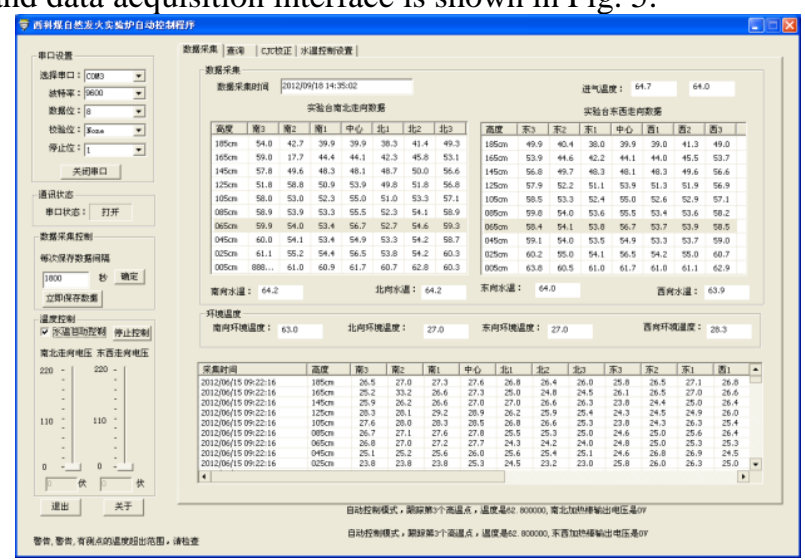

Fig. 5. The monitoring and data acquisition interface.

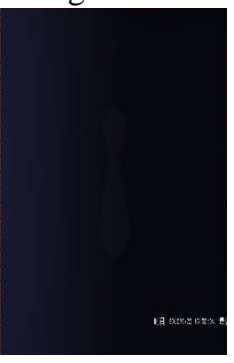

(a)

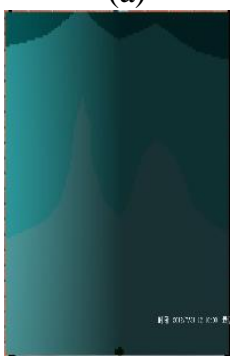

(f)

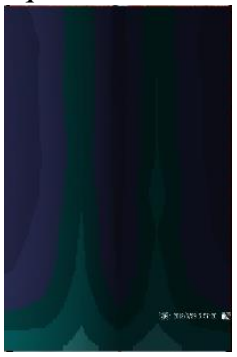

(b)

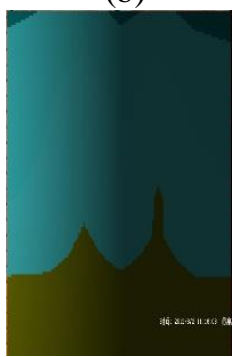

(g)

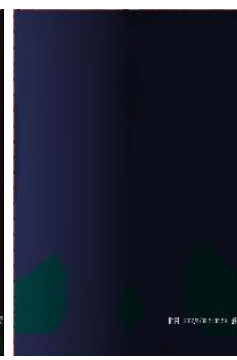

(c)

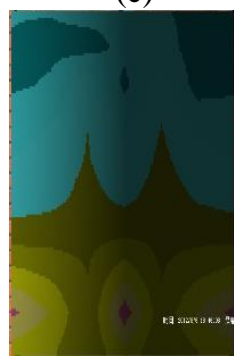

(h)

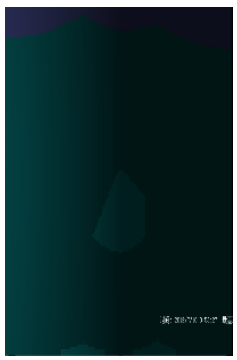

(d)

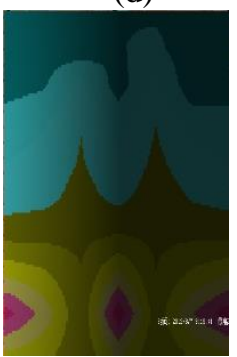

(i)

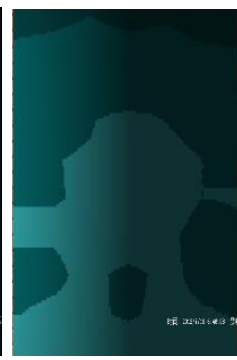

(e)

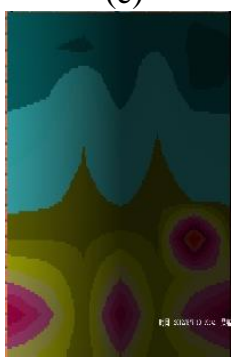

(j) 
Fig. 7 The change of temperature in the furnace of the 3\# coal sample of Ren Jiazhuang mine. The time from (a) to (j) are: (a)2018/6/22 15:52:04 (b)2018/6/29 5:57:20 (c) 2018/6/30 5:02:59 (d) 2018/7/10 3:53:27 (e) 2018/7/11 3:53:27 (f) 2018/7/31 12:16:08 (g) 2018/8/2 $11: 16: 08$ (h) 2018/8/6 21:46:08 (i) 2018/8/7 7:19:41 (j) 2018/8/7 10:19:41

\section{CONCLUSION}

This paper has developed a three-dimensional simulation experimental platform for coal spontaneous combustion, which has the advantages of both numerical simulation and physical simulation. The researchers can easily create virtual coal spontaneous combustion models on the platform, and observe the results of physical simulation and numerical simulation in three-dimensional visualization. It saves the research cost, shortens the study cycle and promotes the study of coal spontaneous combustion mechanism.

\section{ACKNOWLEDGMENT}

This work was supported in part by the National Natural Science Foundation of China under Grant 51974236, in part by the case base project and flipped classroom project of Xi'an University of science and technology.

\section{REFERENCES}

[1] D. Schmal, J. H. Duyzer, and J. W. V. Heuven. "A Model for the Spontaneous Heating of Coal." Fuel 64.7(1985):963-972.

[2] B. Kevin, S.Nicoloas, G. David. "Critical temperatures of some Turkish coals due to spontaneous combustion." Journal of Mines, Metals \& Fuels 36(1988):434-436.

[3] J. W. Zhang, W. Choi, T. Ito, et al. "Modelling and parametric investigations on spontaneous heating in coal pile." Fuel 176(2016): $181-189$.

[4] C. K. Lei, J. Deng, K. Cao, et al. "A random forest approach for predicting coal spontaneous combustion." Fuel 223(2018): 63 - 73.

[5] W. Liu, Y. P .Qin. "Multi-physics coupling model of coal spontaneous combustion in longwall gob area based on moving coordinates." Fuel 188(2017):553-566.

[6] M. Young, "Mathematic Model for Calculating the Shortest Coal Spontaneous Combustion Time." Journal of China Coal Society(2001)26, 516-519.

[7] Y. T. Liang, H. Z. Luo. "An experimental modeling of coal self-heating under low temperature oxidation conditions." Journal of China Coal Society 35(2010)956-959

[8] W. Deng, X. X. Zhong, J. Gu, X.Y Qi, "Changes in Active Functional Groups during Low-Temperature Oxidation of Coal." Mine science and technology 20(2010)35-40.

[9] Yu, Z. J. , Wen, H. , \& Wang, C. . (2019). Numerical Simulation of the Coal Spontaneous Combustion Dangerous Area in Multi-seam Longwall Gobs. Proceedings of the 11th International Mine Ventilation Congress.

[10] W. Liu,, Y. Qin . "Multi-physics coupling model of coal spontaneous combustion in longwall gob area based on moving coordinates." Fuel 188(2017):553-566.

[11] J. C. Xu ,H. Wen, J. Deng, and X. H. Zhang Study Limit Parameter of Coal Self-ignite Fire Safety Science 9(2000):15-17.

[12] J. Deng, J. C. Xu , and H. Q. Wang . "Numerical simulation study on the spontaneous combustion process of the column coal sample." Journal of Liaoning Technical University 90.12(2002):505-509. 